\title{
Magnetic circuit optimization of linear dynamic actuators
}

\author{
Laszlo Kazup ${ }^{1}$, Angela Varadine Szarka ${ }^{1}$ \\ ${ }^{1}$ Reseach Institute of Electronics And Information Technology, University of Miskolc, Miskolc, Hungary
}

\begin{abstract}
Contactless braking methods (with capability of energy recuperation) are more and more widely used and they replace the traditional abrasive and dissipative braking techniques. In case of rotating motion, the method is trivial and often used nowadays. But when the movement is linear and fast alternating, there are only a few possibilities to break the movement. The basic goal of research project is to develop a linear braking method based on the magnetic principle, which enables the efficient and highly controllable braking of alternating movements. Frequency of the alternating movement can be in wide range, aim of the research to develop contactless braking method for vibrating movement for as higher as possible frequency. The research includes examination and further development of possible magnetic implementations and existing methods, so that an efficient construction suitable for the effective linear movement control can be created. The first problem to be solved is design a well-constructed magnetic circuit with high air gap induction, which provides effective and good dynamic parameters for the braking devices. The present paper summarizes the magnetostatics design of "voice-coil linear actuator" type actuators and the effects of structure-related flux scattering and its compensation.
\end{abstract}

\section{Section: RESEARCH PAPER}

Keywords: Magnetic brake; linear brake; magnetic circuit calculation; dynamic braking

Citation: Laszlo Kazup, Angela Varadine Szarka, Magnetic circuit optimization of linear dynamic actuators, Acta IMEKO, vol. 10, no. 3, article 19, September 2021, identifier: IMEKO-ACTA-10 (2021)-03-19

Section Editor: Lorenzo Ciani, University of Florence, Italy

Received February 5, 2021; In final form April 27, 2021; Published September 2021

Copyright: This is an open-access article distributed under the terms of the Creative Commons Attribution 3.0 License, which permits unrestricted use, distribution, and reproduction in any medium, provided the original author and source are credited.

Funding: The described article/presentation/study was carried out as part of the EFOP-3.6.1-16-2016-00011 "Younger and Renewing University - Innovative Knowledge City - institutional development of the University of Miskolc aiming at intelligent specialisation" project implemented in the framework of the Szechenyi 2020 program. The realization of t this project is supported by the European Union, co-financed by the European Social Fund.

Corresponding author: Laszlo Kazup, e-mail: laszlo.kazup@eiki.hu

\section{INTRODUCTION}

In the field of manufacturing, many methods of lifetime testing are used. In case of electronic devices, the stress- and lifetime testing is quite simple in most cases compared to the mechanical tests, when mechanical load emulation is very difficult and expensive. For example, power tools are tested with different loads and some of them have alternating linear movements which should be loaded. There are not fully developed contactless load emulating methods for this application. Sometimes hydraulic system is used with low efficiency. In case of traditional practice test methods, an operator works with the device under test (DUT), he/she cuts, sands, or planes different materials. This type of testing is expensive, not reliable, and less repeatable than the automated test solutions. In some tests the operators were replaced by industrial robots, but the test is still expensive and dirty due to the using of real materials. The best result would be a system which can emulate the loading force without any physical contact, abrasion, and dirt. Research of braking method for fast alternating linear movements by using contactless magnetic methods is in the focus of our project. This work is the second phase of the research and development project, which was started in 2008. The original goal of the project was to develop a special magnetic brake which can simulate the real operation of an electric jig saw to replace the traditional practice test method in which operators perform the full test process by cutting different types of material such as wood, steel, aluminium, etc. The repeatability of this test method is very low, as well as the reliability of the documented test circumstances. Also a lot of waste and dirt in the test centre was produced. In those days a special hydraulic brake was developed in Switzerland to solve this problem, but its performance, controllability and reliability was poor. The analysis of different dynamic brake constructions and methods have proved that the most reliable and efficient solution can be achieved by using a magnetic construction. The test equipment should perform a special braking characteristic which can be controlled even in a single moving period, and in case of using an electromagnetic solution, these properties can be 
realized. The development of this test equipment needed both practical and theoretical improvement. The prototype testing and evaluation originated the further hypotheses. Our research group aims to create general theoretical and modelling methodology supporting reliable practical realization of dynamically controlled magnetic fields which can be used in many different industries to optimize the performance of voice-coil type linear actuators and brakes. My research work includes systematic analysis of all possible and realistic magnetic circuit constructions, optimization of the magnetic circuits, and also performing static and dynamic simulations to maximize the efficiency. The research includes analysis of voice-coil type magnetic actuators, design of magnetic circuit to maximize efficiency and reliability and minimize weight and size of the brake. The first part of this paper includes introduction of a method for transformation of 2-dimension magnetic calculations to the cylindrical coordinate system and presents the analysis of the flux leakage and its effects to the results of calculations. The calculations are confirmed by finite element simulations, and the results are also used to correct the differences caused by the flux leakage. These calculations, simulations and corrections were solved for different shapes to realize a shape- and size independent model to calculate the correct average flux density in the air gap. The second part of paper presents the results of dynamic simulations, by which dynamic behaviour (relationship between the current and the force in dynamic cases, eddy current- and solid losses, etc.) of the voice coil-type actuator is analysed.

This research is the extension of the air-gap induction distortion analysis, which work is described in paper "A. Diagnostics of air-gap induction's distortion in linear magnetic brake for dynamic applications", [1] [3].

\section{DESIGN OF CYLINDRICAL MAGNETIC CIRCUIT WITH TWO- DIMENSIONAL, PLANE CROSS-SECTION MODEL CALCULATIONS}

The aim of the first part of the work was to theoretically establish, develop, and validate a method that transforms the dimensions of a cylindrical symmetrical magnetic circuit of a given size into an equivalent two-dimensional cross-sectional and constant depth model. In this way, cylindrical magnetic circuits can also be calculated. In this method the cylindrical magnetic circuit is "spread out" so that the vertical ( $\mathrm{z}$ ) dimensions are

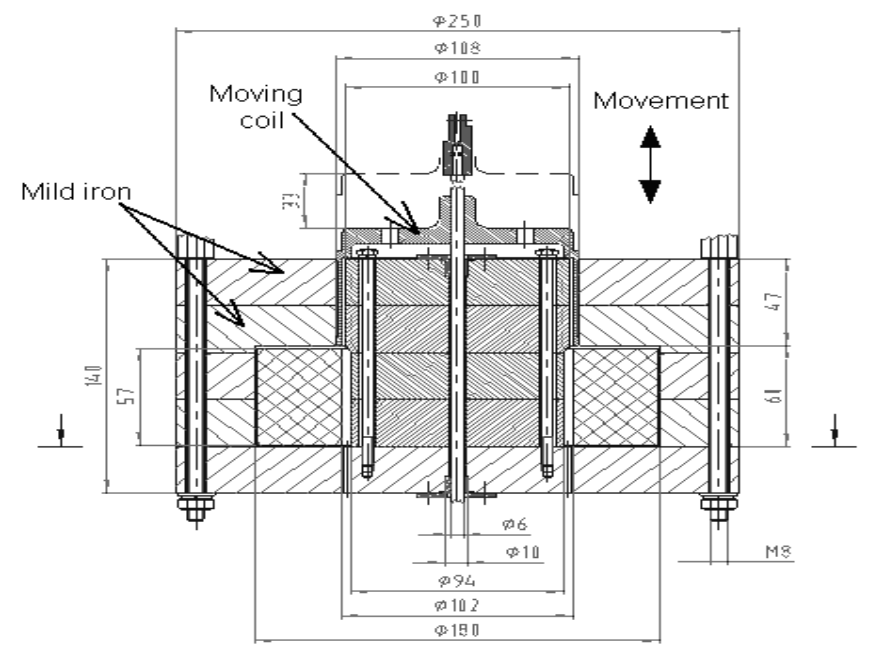

Figure 1. The mechanical drawing of the first experimental dynamic magnetic brake prototype, [3]. leaved unchanged, and the $r$ values are transformed into $x$ values to create a flat-section, fixed-depth model ("cubic model") in which the volume of each part is the same as the volume of the corresponding parts in the cylindrical model, so the two models is connected to each other by the unchanged value of the flux. However, inductions calculated in the planar model is also valid for the transformation, the calculated values correspond to the average values in the cylindrical model, since values of the magnetic induction in the cylindrical model are changing in radial direction. As a result, higher induction values are observed on the inner half of the cylindrical parts and lower on the outer half. Figure 1. shows the dimensions of a typical cylindrical model, and Figure 2 illustrates the corresponding $x$ and $z$ dimensions in a planar cross-sectional model for transformation. [6], [7]

The depth $d$ of the plane cross-section fixed-depth model should be taken so that the $x$ dimensions of the plane model are close to the radius differences of the cylindrical model in the area most affected by the test (this is practically the air gap). Thus, for practical reasons, depth dimension $d$ is selected equal to the length of line at the air gap center circle which is as follows:

$$
d=\left(r_{2}+r_{3}\right) \pi
$$

To determine the relationship between the radius and the $x$ values, the volume equality was used as already mentioned above, which is the following:

$$
V_{n_{r h}}=V_{n_{x y}}\left(r_{n}^{2}-r_{n-1}^{2}\right) \pi h_{m}=x_{n} y_{m} d \text {. }
$$

Since the given $b$ and $y$ values in the two models are the same based on a previous condition, the final correlation for the $x$ values after transformations is as follows:

$$
x_{n}=\frac{\left(r_{n}{ }^{2}-r_{n-1}{ }^{2}\right) \pi}{d} \text {. }
$$

In the above relation if $n=1$, then $r$ is 0 , assuming that inner bar (iron cores and also magnets) is cylindrical. If the inner bar has ring section, value of $r_{0}$ is equal to radius of inner ring.

\section{VERIFICATION OF THE RELATIONSHIPS RECEIVED BY FINITE ELEMENT SIMULATION}

To verify transformation relationships introduced above, a transformation of a cylindrical magnetic circuit of a given size was performed. In determining the depth dimension $d$, the length

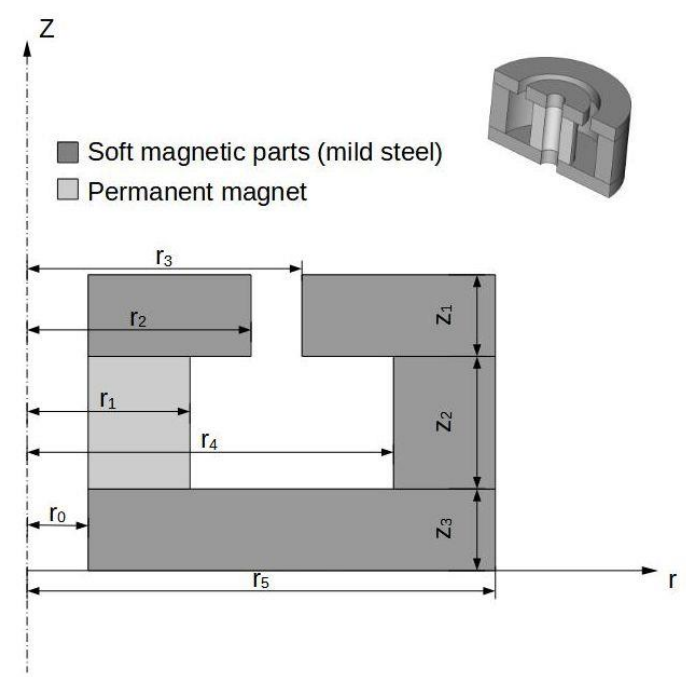

Figure 2. The half cross-section of a typical cylindrical magnetic circuit. 
Table 1. Basic dimensions of the cylindrical model and calculated dimensions of the transformed model.

\begin{tabular}{|c|c|c|}
\hline Parameter & Description & Value $(\mathrm{mm})$ \\
\hline \multicolumn{3}{|c|}{ Original values of the cylindrical model } \\
\hline$r_{0}$ & Internal radius of the ring magnet & 40 \\
\hline$r_{1}$ & External radius of the ring magnet & 70 \\
\hline$r_{2}$ & Internal radius of the air gap & \\
\hline$r_{3}$ & External radius of the air gap & 72.5 \\
\hline$r_{4}$ & Internal radius of the outer ring & 130 \\
\hline$r_{5}$ & External radius of the outer ring & 150 \\
\hline$z_{1}$ & Height of the air gap & 20 \\
\hline$z_{2}$ & Height of the magnet/outer ring & 60 \\
\hline$z_{3}$ & Height of the bottom cylinder & 20 \\
\hline \multicolumn{3}{|c|}{ Transformed values } \\
\hline$x_{1}$ & Width of the transformed magnet & 11.23 \\
\hline$x_{2}$ & $\begin{array}{l}\text { Distance between the air gap and the } \\
\text { magnet }\end{array}$ & 23.16 \\
\hline$x_{3}$ & Air gap length & $2.49998(\sim 2.5)$ \\
\hline$x_{4}$ & $\begin{array}{l}\text { Distance between the air gap and the } \\
\text { outer mild iron part }\end{array}$ & 81.71 \\
\hline$x_{5}$ & $\begin{array}{l}\text { Width of the outer mild iron part } \\
\text { (transformed one of the original outer } \\
\text { ring) }\end{array}$ & 39.3 \\
\hline$z_{1}$ & Height of the air gap & 20 \\
\hline$z_{2}$ & $\begin{array}{l}\text { Height of the magnet/ outer mild iron } \\
\text { part }\end{array}$ & 60 \\
\hline$z_{3}$ & Height of the bottom mild iron part & 20 \\
\hline$d$ & Depth of the magnetic circuit & 447.68 \\
\hline
\end{tabular}

of the central circle of the air gap was considered, which will result nearly equal air gap induction in plane model and in the cylindrical model. The dimensions of the initial cylindrical model and the plane model which is the result of the transformation are summarized in Table 1.

The next step was to determine the air gap induction based on static magnetic calculations in which dimensions of the plane model were used. Initial data had to be determined, these are the main magnetic parameters of the applied soft ferromagnetic and permanent magnetic material. These data were received from the finite element simulation software database for better comparison. These material properties are as follows (the operating point values of the magnet have been determined graphically):

- Permanent magnet

- Material: Y25 ferrite magnet

- Residual induction: $0.378 \mathrm{~T}$

o Coercive force: $153035 \mathrm{~A} / \mathrm{m}$

- Relative permeability at the operating point section of the demagnetization curve: 1.9

- $\quad$ Soft steel parts
- Material: 1010 low carbon soft steel
- Relative permeability: $\sim 10^{3}$
- Maximum induction: $1 \mathrm{~T}$ (in linear part of $B$ - $H$ graph)

The summarized relative permeability of the permanent magnet and the air gap is three order of magnitude smaller than relative permeability of the body, therefore the permeability of the soft iron was neglected in the calculations. In this phase of the research the magnetic calculations focused to the permanent magnet and the air gap. Based on the above described initial data, the main steps of the calculations were as follows.
1.) Determining the operating point of the magnet by equation of the air gap line (based on data of the transformed geometry):

$$
\begin{aligned}
& H_{m}=\frac{-1}{\mu_{0}} \cdot \frac{x_{1}}{y_{1}} \cdot \frac{x_{3}}{y_{2}} \cdot B_{m} \\
& H_{m}=-7000 \frac{\mathrm{A}}{\mathrm{m}} \\
& B_{m}=0.3726 \mathrm{~T}
\end{aligned}
$$

2.) Determination of the flux:

$$
\begin{aligned}
& \Phi=B \cdot A=B_{m} \cdot A_{m}=B_{m} \cdot x_{1} \cdot d \\
& =1.873 \cdot 10^{-3} \mathrm{~Wb} .
\end{aligned}
$$

3.) Determination of the air gap induction:

$$
B=\frac{\Phi}{A} \rightarrow B_{\delta}=\frac{\Phi}{A_{\delta}}=\frac{\Phi}{y_{1} d}=0.2092 \mathrm{~T} .
$$

After performing the calculations, both the original cylindrical and the transformed magnetic circuits were simulated by FEMM 4.0 finite element simulation software [7]. The simulation results and the calculated values are summarized in Table 2 .

The air gap induction value was approx. $75 \%$ compared to the calculated one in the simulation (the reason is the leakage flux around the air gap). In spite of this, the average value of the induction in the magnet and the flux of the magnetic circuit showed only a very small difference from the calculated data. According to these results we can state that the model and transformation provided good results and can be used in the next stage of the research. Most of the differences from the simulated values (the finite element simulation is the validation of the calculations in this stage) are due to the leakage flux, correction of which needs further studies. Although flux in the air gap is also $72 \%$ less, including leakage induction lines, simulation gives the original calculated flux value. The further paragraphs show that in case of flux leakage compensation the calculations will approximate the simulated results very close.

\section{CALCULATION OF MAGNETIC CIRCUIT FOR REQUIRED AIR GAP INDUCTION AND AIR GAP DEPTH}

While the previous calculations illustrated the transformation of a magnetic circuit with given dimensions, in practice, developing a so-called "voice-coil-actuator", a much more common problem is adjusting dimensions of the structure, especially dimensions of the magnet to be used, to the given air gap height and air gap induction value. In such a case, the minimum air gap diameter has to be defined at which the given induction can be performed at the specified air gap height.

Also, if the diameter of the air gap is fixed, feasibility of the desired induction with the given permanent magnet type at the specified sizes should be checked. In addition, the effect of

Table 2. Comparison of the calculated and the simulated results

\begin{tabular}{cccc}
\hline Parameter & $\begin{array}{c}\text { Calculated } \\
\text { value }\end{array}$ & $\begin{array}{c}\text { Simulated value } \\
\text { (cylindrical model) }\end{array}$ & $\begin{array}{c}\text { Simulated value } \\
\text { (plane model) }\end{array}$ \\
\hline$B_{\mathrm{m}}$ & $0.3726 \mathrm{~T}$ & $0.3739 \mathrm{~T}$ & $0.3744 \mathrm{~T}$ \\
$\Phi_{\mathrm{m}}$ & $1.873 \cdot 10^{-3} \mathrm{~Wb}$ & $1.879 \cdot 10^{-3} \mathrm{~Wb}$ & $1.886 \cdot 10^{-3} \mathrm{~Wb}$ \\
$B_{\delta}$ & $0.2092 \mathrm{~T}$ & $0.1509 \mathrm{~T}$ & $0.138 \mathrm{~T}$ \\
$\Phi_{\delta}$ & $1.873 \cdot 10^{-3} \mathrm{~Wb}$ & $1.351 \cdot 10^{-3} \mathrm{~Wb}$ & $1.24 \cdot 10^{-3} \mathrm{~Wb}$ \\
\hline
\end{tabular}


leakage flux must be considered when calculating these data (the study and correction of leakage is discussed in chapter VI.

\subsection{Calculation steps}

1.) Definition of operating point values of magnets

The real demagnetization curve of permanent magnets is linear in a relatively wide range, it has nonlinearity only near the coercive force. Therefore, the operating point of the magnet should be defined to provide maximum value of the product $B \cdot H$, which is in the point $B_{m}=B_{r} / 2$ in the practice.

2.) Determination of the cross section of magnets perpendicular to flux

The air gap flux can be determined from the air gap cross section and the desired induction. Since the air gap and the flux of the magnet are the same in theory, the cross section of the magnet can be determined from the equation $\Phi=B \cdot A$. The value of the radius of this surface must be checked to ensure that it is smaller than the inner circle line of the air gap (in the case of a plane model the test can also be done, in which case the $x$ value at the beginning of the air gap must be greater than or equal to $x)$.

If the evaluation shows that the desired induction is not feasible at a given air gap circle, the air gap diameter must be chosen to be higher, or if it is not possible, the magnet can be used with a different (higher) induction than the optimal operating point, which will result an increase in the length of the magnet (see point 3).

In practice, the air gap and the flux of the magnet do not match due to scattering, so the correction described later should be applied.

3.) Determination of the optimal length of permanent magnets

Since the reluctance of the iron body is neglected according to an earlier condition, the equation $\oint H d l$ in the magnetic circuit can be defined as follows (without considering leakage correction):

$$
H_{\delta} \delta+H_{m} y_{m}=0, \quad \frac{B_{m}}{\mu_{0} \mu_{m}} y_{m}=\frac{-B_{\delta}}{\mu_{0}} \delta
$$

where $\mu_{m}$ is the relative permeability of the permanent magnet (typically will be between 1 and 2), $B_{m}$ is the operating point induction of the permanent magnet, $y_{m}$ is the length of permanent magnet to be defined, $B_{\delta}$ required induction, $\delta$ length of air gap.

Calculation example shows that the required air gap induction can be achieved without operating the magnet at the operating point. The demagnetizing field strength is less than the operating point field strength, but in this case the magnet length must be greater than the optimal value for the equation to be realized.

If the goal is to use a permanent magnet with the smallest possible volume (and at the same time the lowest cost), it is advisable to use the optimal dimensions determined by the operating point. To achieve this, custom-made permanent magnets are necessary in practice. Experience shows that when using commercial permanent magnets from catalogs some we should accept some comptonization.

4.) Determination of cross section of soft iron body

Based on the data sheets of various commonly used soft iron materials, we can state that approx. up to $1 \mathrm{~T}$, their $B-H$ curve is linear, so it is not recommended to design above this value.
Otherwise, especially near saturation, the relative permeability of the iron decreases, and in this case the reluctance of the given section is no longer negligible. [6]

The previously determined flux value and the maximum induction can be calculated based on the cross-sectional dimensions.

\section{APPLICATION OF FERRITE MAGNETS IN THE OUTER RING AS A FLUX CONDUCTOR}

The results of the dynamic simulations proved, that the reluctance of the magnetic circuit and the properties of the materials in the vertical columns and rings greatly influence the value of the inductivity of the moving coil and the magnitude of the eddy current losses during dynamic operation. We have also examined an initial experimental design that included a permanent magnet both inside and outside. A static study of this construction was also carried out, during which it was found that the external permanent magnet does not substantially increase the air gap induction in the magnetic-air gap-magnet series magnetic circuit, it only increases the demagnetization field strength of the inner, so-called "working" magnet, which results operating point down shifting of this magnet.

However, dynamic studies have discovered some advantages, which are the reduced inductance of the moving coil and reduced power dissipation of iron loss. Results show that the inductance is approx. half of the level when using soft iron instead of an external magnet in such a structure. The conclusion is that if the dimensions of the external magnet are determined so that the magnetic field strength inside of it is close to 0 , then the magnetic ring behaves as a "flux conductor" like iron with low relative permeability.

This operating state is also characteristic of soft iron materials in the near-saturation state, with significant flux leakage. However, in the case of permanent magnets, the leakage is minimal. The results are better dynamic parameters caused by reduced inductance of the moving coil as well as reduced eddy current losses, but in turn it and does not reduce the performance of the correctly calculated dimensions working magnet.

If this solution is used for the sizing of the magnetic circuit, the last point of the design steps is modified as follows: the cross section of the external magnetic ring at which the induction is equal to the value of the residual induction of the magnet must be determined. For practical reasons, it is recommended to choose the length of the external magnet as equal to the length of the inner magnet (this simplifies the construction).

While experience show that neodymium iron-boron (or samarium cobalt at higher operating temperatures) is the most suitable material for the internal working magnet due to its high energy density, conventional strontium ferrite magnets can also be used for the external magnetic ring used as a flux conductor. They have lower prices than the two types of magnets listed above and, since they are used as external elements, their relatively large size is not limited by critical parameters affecting the moving mass, such as the diameter of the moving coil.

\section{ANALYSIS AND CORRECTION OF FLUX LEAKAGE}

The leakage of magnetic induction lines in a magnetic circuit with an air gap is a complex problem that depends on several design and operating parameters. Practical experiences show that in optimal situation more than $90 \%$ of the leakage flux is present around the air gap, but in case of permanent magnets operating out of the optimal operating point or at soft iron sections near 
the saturation, significant part of lines may close outside the magnetic circuit. There are several estimation work-help documents of leakage calculations, including air-gap leakage calculations are available from permanent magnet manufacturers to magnetic circuit designers. In these documents, the air gap and the field around it are divided into several areas depending on the type of magnetic circuit, which can include semicylindrical, semispherical, quarter-spherical and/or prismatic areas. The magnetic reluctances of these parallel fields are defined using exact, empirical formulas. However, these definitions help only in the design of certain magnetic circuits with frequently used structures. [9], [10]

In general cases, the effects of leakage in an arbitrary magnetic circuit can be estimated most accurately by finite element simulation. This requires exact and accurate information about geometric model and characteristics of the applied materials.

In order to make method outlined earlier applicable in practice for design "voice-coil actuator" type electromagnetic actuators, correct levels of leakage flux should be estimated. In the first step we have worked out an algorithm for automated static magnetic calculations. The calculations are based on the basic magnetostatic calculations mentioned in Chapter 4.

The main input parameters of this algorithm are the residual induction of the applied magnet types (internal and external), its coercive force, the height of the air gap, and the radius of its internal and external sections. The required air gap induction can be given by the following values:

start value

stop value

number of steps

The algorithm can generate a table including the most important geometric parameters of the parametric simulation sequences, which are the following:

$r_{0}$ : the inner radius of the inner magnet

$r_{1}$ : the outer radius of the inner magnet, equal to the inner radius of the air gap

$r_{2}$ : the inner radius of the outer magnet, equal to the outer radius of the air gap

$r_{3}$ : the outer radius of the outer magnet

Several simulation series were performed to analyze the effect of flux leakage and determine a correction relationship. These simulations were built by using ANSYS MAXWELL 2D finite element simulation software [8]. The models in the simulation were axis-symmetrical models. In the first simulation a magnetic circuit according to Figure 1. was used, in which the radius of the central circle of the air gap is $70 \mathrm{~mm}$ and the height of the air gap is $20 \mathrm{~mm}$. The start value of induction is $0.1 \mathrm{~T}$ and the stop value is $1 \mathrm{~T}$, with $0.05 \mathrm{~T}$ steps. The $1 \mathrm{~T}$ stop value is maximized by the given air gap center circle radius. Using the received geometric dimensions, a parametric simulation was prepared to investigate the difference between the average induction value experienced in the air gap and the initial air gap induction value due to flux leakage.

In the simulation deviation of working range from the operating point was examined for internal magnets (induction value is $0.67 \mathrm{~T}$ for $\mathrm{N} 35$ type magnets) and deviation of the average induction from the residual induction value $\left(B_{\mathrm{r}}=0.38 \mathrm{~T}\right)$ was analyzed. The cross-sections of the soft iron elements of the construction were set large enough to avoid saturation or close to saturation operation. Initial values and the results of the simulation are summarized in Table 3.

Simulation results prove slight increase (between $72 \%$ and $78 \%)$ in the ratio of theoretical and simulated air gap induction
Table 3. Comparison of the calculated and the simulated results

\begin{tabular}{lcccc}
\hline $\begin{array}{c}\text { Required } \\
\text { air-gap } \\
\text { induction } \\
\text { in T }\end{array}$ & $\begin{array}{c}\text { Simulated } \\
\text { air-gap } \\
\text { induction } \\
\text { in T }\end{array}$ & $\begin{array}{c}\text { Ratio } \\
\text { simulated } \\
\text { vs. required } \\
\text { induction } \\
\text { in \% }\end{array}$ & $\begin{array}{c}\text { Operating } \\
\text { point } \\
\text { induction of } \\
\text { inner magnet } \\
\text { in T }\end{array}$ & $\begin{array}{c}\text { Operating } \\
\text { point } \\
\text { induction of } \\
\text { external } \\
\text { magnet in T }\end{array}$ \\
\hline 0.1 & 0.071 & 71.00 & 0.793 & 0.335 \\
0.15 & 0.109 & 72.66 & 0.784 & 0.344 \\
0.2 & 0.147 & 73.50 & 0.779 & 0.351 \\
0.25 & 0.185 & 74.00 & 0.774 & 0.356 \\
0.3 & 0.223 & 74.33 & 0.770 & 0.362 \\
0.35 & 0.261 & 74.57 & 0.767 & 0.367 \\
0.4 & 0.299 & 74.75 & 0.764 & 0.371 \\
0.45 & 0.338 & 75.11 & 0.761 & 0.375 \\
0.5 & 0.376 & 75.20 & 0.758 & 0.379 \\
0.55 & 0.415 & 75.45 & 0.755 & 0.382 \\
0.6 & 0.453 & 75.50 & 0.752 & 0.386 \\
0.65 & 0.492 & 75.69 & 0.750 & 0.389 \\
0.7 & 0.532 & 76.00 & 0.746 & 0.393 \\
0.75 & 0.571 & 76.13 & 0.743 & 0.395 \\
0.8 & 0.611 & 76.37 & 0.740 & 0.399 \\
0.85 & 0.651 & 76.59 & 0.736 & 0.402 \\
0.9 & 0.692 & 76.88 & 0.732 & 0.405 \\
0.95 & 0.734 & 77.26 & 0.727 & 0.409 \\
1 & 0.778 & 77.80 & 0.717 & 0.413 \\
\hline & & & &
\end{tabular}

when varying the value of the air gap induction from $0.1 \mathrm{~T}$ to $1 \mathrm{~T}$.

Examining the simulation results, we can find that that the operating point of the working magnet on the demagnetization curve $B-H$ always shifts to the right of the ideal operating point. This phenomenon is caused by the modelling error, that is the computational models do not consider either the alternative reluctances caused by leakage or the real magnetization curve of the body. However, the difference in practice is small enough to be neglected in the general case. For this reason, the operating point of the external magnets also differs slightly from $H=0$.

The next step of the work is to determine a correction factor for the required initial air gap induction, resulting corrected geometric parameters at which the value of the simulated air gap induction will be equal to the originally required (not corrected) air gap induction. The correction relationship determined from the simulation results is expressed by the following equation

$$
B_{\delta_{\mathrm{korr}}}=B_{\delta} \cdot 1.281+0.018 \text {. }
$$

Including this correction into the original algorithm, the parametric simulation was repeated with initial values of $0.1 \mathrm{~T}$ and $0.75 \mathrm{~T}$. The higher values is selected according to the maximum possible $1 \mathrm{~T}$ corrected initial value of induction. The results are shown in Table 4.

\section{THE EFFECT OF PERMANENT MAGNETS USED AS FLUX CONDUCTORS ON DYNAMIC BEHAVIOUR}

Experience has shown that the method mentioned in the previous chapter, discussing the use of permanent magnets instead of mild steel in the outer ring of cylindrical magnet circuits, improves the dynamic behaviour of such magnetic actuators. This improvement is detectable in both permanent magnet and electromagnetically excited constructions.

Due to the structure of the above-mentioned constructions, the magnetic field created by the vertically arranged voice coil current has an impact on the magnetic field of the stator (the still 
Table 4. Comparison of the calculated and the simulated results

\begin{tabular}{ccl}
\hline $\begin{array}{c}\text { Required } \\
\text { induction } \\
\text { in T }\end{array}$ & $\begin{array}{c}\text { Corrected value of } \\
\text { induction for } \\
\text { simulation input in T }\end{array}$ & $\begin{array}{c}\text { Simulated } \\
\text { induction } \\
\text { in T }\end{array}$ \\
\hline 0.1 & 0.139 & 0.106 \\
0.15 & 0.205 & 0.154 \\
0.2 & 0.271 & 0.202 \\
0.25 & 0.337 & 0.251 \\
0.3 & 0.402 & 0.3 \\
0.35 & 0.468 & 0.35 \\
0.4 & 0.533 & 0.399 \\
0.45 & 0.598 & 0.449 \\
0.5 & 0.663 & 0.499 \\
0.55 & 0.728 & 0.55 \\
0.6 & 0.792 & 0.6 \\
0.65 & 0.857 & 0.652 \\
0.7 & 0.919 & 0.705 \\
0.75 & 0.984 & 0.759 \\
\hline
\end{tabular}

part of the actuator, which means the overall magnetic circuit) as well. Previous tests and simulations in the field have verified that in certain applications, when a high current flows through the voice coil, the magnetic field created by the coil modifies, distorts the originally homogeneous magnetic field of the air gap. The distortion of induction in the air gap is particularly high in the case of voice coils excited with direct current. As a result, since the length of the voice coil is finite, during operation the force applied to the turns exceeds the mechanical limits the construction of the voice coil was designed to withstand in the first place. Thus, if the actuator is designed to exert constant or slowly changing high force, this distortion must be taken into consideration when developing the construction of the voice coil. Figure 3 illustrates the character of distortion of induction in the air gap in the case of direct current controlling.

Furthermore, the tests have revealed that since in most cases the mild steel parts of such actuators are made of solid mild steel units, locally induced eddy currents compensate the distortion of induction in the air gap in the case of rapidly changing, dynamic voice coil currents. As indicated by tests on the first, electromagnetically excited prototype of the braking system to be developed, even in case of $20 \mathrm{~Hz}$ current components, the

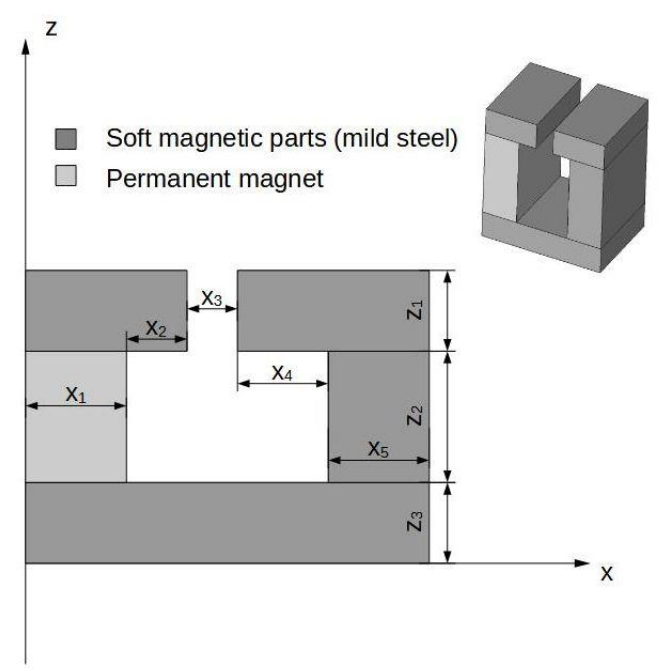

Figure 3. The cross-section of the 2-D model.

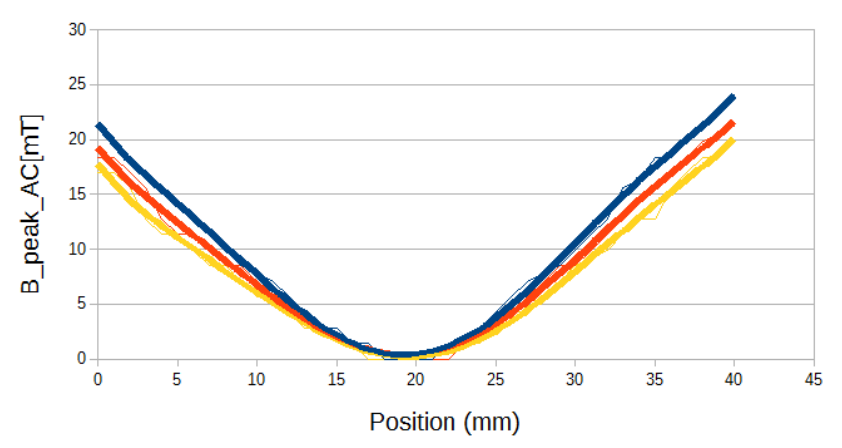

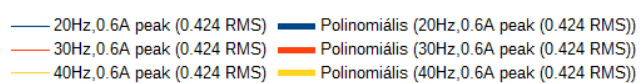

Figure 4. Average AC component of the air gap induction as a function of the frequency in a voice-coil type linear actuator excited by DC current.

distortion of the induction in the air gap considerably decreases as compared to excitation with direct current. The results of a series of such tests are shown in Figure 4.

On the other hand, later finite element simulation tests on further developed magnetic constructions have revealed another problem: the momentarily self-inductance of the voice coil is connected to the reluctance of the magnetic ring of the stator. For dynamic operation, it is essential that the self-inductance of the voice coil be as low as possible in order to achieve the adequate impulse response.

When excitation is carried out with $\mathrm{NdFeB}$ permanent magnets, the self-inductance of the voice coil in the construction examined (a magnetic ring with a $55 \mathrm{~mm}$ middle/mean diameter air gap) is relatively low, approximately $13 \mu \mathrm{H}$, which may be appropriate from the aspect of dynamic behaviour. In this case, finite element simulations have resulted in a linear connection with a fairly good approximation between the current in the coil and the force generated in the coil. This is caused by the high reluctance of the magnetic ring in the environment of the voice coil, for the magnetic field of the voice coil can considerably influence the volume and direction of inductance in permanent

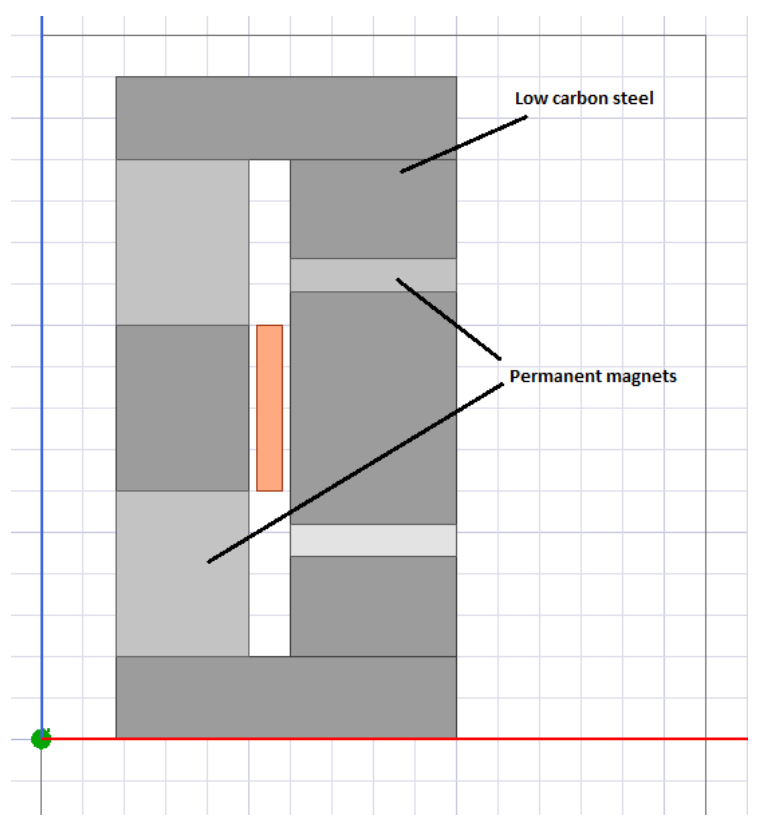

Figure 5. The simulation model of an improved construction of an NdFeBmagnet based voice coil actuator (brake) including permanent magnets in the outer ring. 
magnets only in case of a much higher value of excitation than the optimal operational value in the bias point, due to the character of demagnetization curve of the permanent magnet. However, further dynamic simulations indicate that with the help of the flux conductor solution, discussed in the previous chapter, the self-inductance of the voice coil can be decreased even more (about $10 \%-12 \%$ ). Figure 5 demonstrates the a forementioned construction solution, while the relevant results of the finite element simulations are shown in Figure 6 and Figure 7.

The dynamic analysis of the construction including permanent magnets has shown that this buildup can have quite excellent dynamic behaviour even without extra magnets. However, in the case of electromagnetic excitation, the entire magnetic ring is by default made of mild steel of high relative permeability, owing to which the reluctance of the magnetic ring is low and the magnetic field generated locally due to the current in the voice coil causes greater distortion in the original magnetic field of the stator excited with current pulse / stator supplied with excitation current. Result of a finite element simulation on an electromagnetically excited construction (the size of the buildup is the same) indicate that in this case the self-inductance of the voice coil (identical in the case of voice coil dimensions) is about 100 times higher than the same value of the $\mathrm{NdFeB}$ magnet-based construction, and the exciting electric current changes considerably and non-linearly between the average value and depending on time. This simulation result is shown on Figure 8. The reason for this is that the greater part of the metallic body is magnetized to almost full saturation for the sake of the greatest air gap induction possible, and in certain sections, due to the magnetic field of the voice coil, the temporary operating point shifts to the non-linear part of the magnetization curve. This results in a non-linear connection between the voice coil current and the force generated, which, together with a highvalue induction impair the dynamic behaviour of the construction. If certain parts of the outer ring of the stator consist of permanent magnets with the operation point settings defined in the previous chapter, this non-linearity and the inductance of the voice coil may be decreased significantly, thus improving dynamic behaviour.

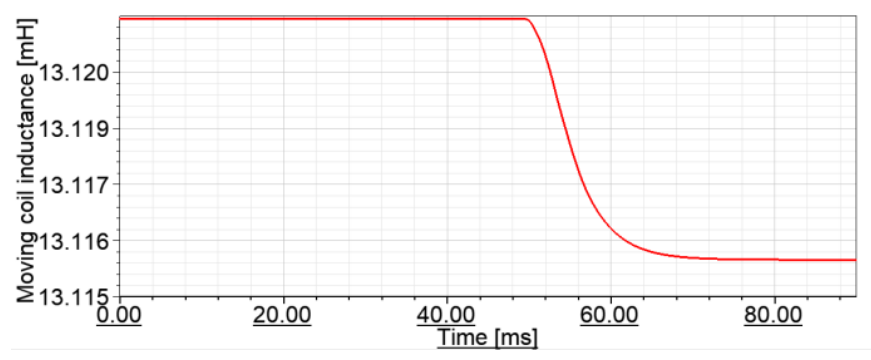

Figure 6. Moving coil inductance of the original construction (current flow starts at $50 \mathrm{~ms}$ )

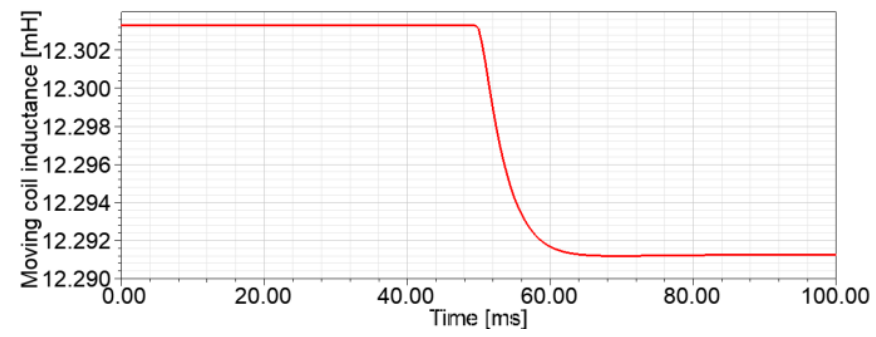

Figure 7. Moving coil inductance of the improved construction (current flow starts at $50 \mathrm{~ms}$ )

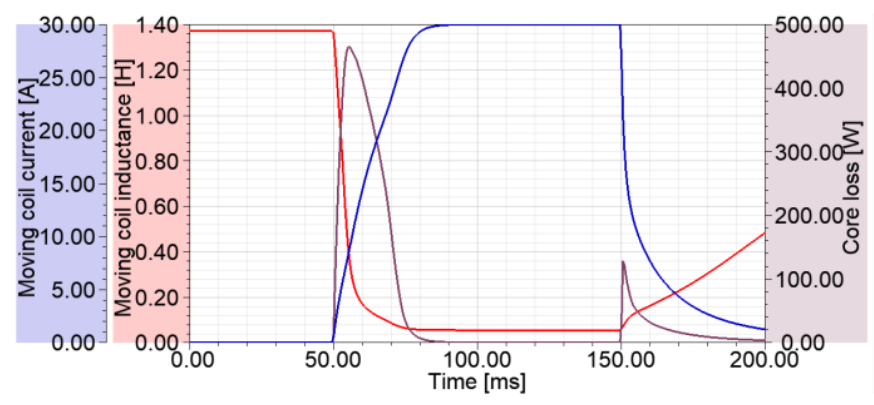

Figure 8. Dynamic finite element simulation of a construction excited by electromagnet

Consequently, the solution discussed in the previous chapter is capable of improving the dynamic behaviour of a so-called "voice-coil-actuator" by decreasing the inductance of the voice coil and its non-linear nature, especially if the excitation of the magnetic ring of the stator is carried out with an electromagnet.

\section{CONCLUSIONS AND OUTLOOK}

Results of the research show that air gap induction correction is an effective method to calculate geometry of magnets and checking the real air gap induction for the calculated geometry for magnetic circuits of so-called "speaker-type voice coil actuator" actuators. Accuracy of air gap induction simulation can be increased if considering further construction details, like join deviations or detailed material properties. Difference between the magnetic properties of real soft magnetic material and simulated material may also cause some simulation error. Converting cylindrical, axially symmetrical magnetic constructions to plane model by defined geometric transformations, the induction and field strength values of the magnetic circuit's sections can be determined with acceptable accuracy. The acceptable accuracy highly depends on the compensation capacity of the control system to be used in the system, therefore checking and correcting the calculations by finite element simulations can be still useful.

Validation of the developed calculation and simulation methods is in progress. A prototype is designed and built with the geometrical dimensions defined by the described methods; tests will be performed in the near future. The validated methods will be used for development and optimization of industrial testing processes.

\section{ACKNOWLEDGEMENT}

This research was supported by the European Union and the Hungarian State, co-financed by the European Regional Development Fund in the framework of the GINOP-2.3.4-152016-00004 project, aimed to promote the cooperation between the higher education and the industry.

\section{REFERENCES}

[1] A. Váradiné Szarka, Linear magnetic break of special test requirements with dynamic performance, Journal of Electrical and Electronics Engineering, Vol. 3, No. 2, 2010, pp. 237-240. Online [Accessed 2 September 2021] https://www.ingentaconnect.com/content/doaj/18446035/201 0/00000003/00000002/art00031

[2] C. H. Chen, A. K. Higgins, R. M. Strnat, Effect of geometry on magnetization distortion in closed-circuit magnetic measurements, Journal of Magnetism and Magnetic Materials, Vol. 320, No. 9, 
2008, pp. $1597-1656$.

DOI: $10.1016 / \mathrm{i} . j \mathrm{mmm} .2008 .01 .035$

[3] L. Kazup, A. Váradiné Szarka, Diagnostics of air-gap induction's distortion in linear magnetic brake for dynamic applications, XXI IMEKO World Congress on Measurement in Research and Industry, Prague, Czech Republic, 30 August - 4 September 2015, pp. 905-908. Online [Accessed 2 Septemner 2021] https://www.imeko.org/publications/wc-2015/IMEKO-WC2015-TC7-190.pdf

[4] G. Kovács, M. Kuczmann, Simulation of a developed magnetic flux leakage method, Pollack Periodica, Vol. 4, No. 2, 2009, pp. 45-56.

DOI: $10.1556 /$ Pollack.4.2009.2.5

[5] M. Kuczmann, Nonlinear finite element method in magnetism, Pollack Periodica, Vol. 4, No. 2, 2009, pp. 13-24.

DOI: $10.1556 /$ Pollack.4.2009.2.2

[6] Nikola A. Spaldin, Magnetic Materials: Fundamentals and Device Applications, Cambridge University Press, 2003, ISBN 9780521016582
[7] Heinz E. Knoepfel, Magnetic Fields, John Wiley \& Sons, 2008, ISBN 3527617426.

[8] FEMM - Finite Element Method Magnetics - Homepage. Online [Accessed 2 September 2021]

https://www.femm.info/wiki/HomePage

[9] ANSYS Maxwell - Product Homepage. Online [Accessed 2 September 2021] https://www.ansys.com/products/electronics/ansys-maxwell

[10] Magnetic Circuit Design Guide - TDK Tech Notes. Online [Accessed 2 September 2021]

https://product.tdk.com/en/products/magnet/technote/design guide.html

[11] Design of Magnetic Circuits - Tokyo Ferrite. Online [Accessed 2 September 2021]

https://www.tokyoferrite-ho.co.jp/en/wordpress/wpcontent/uploads/2017/03/technical 02.pdf 